\title{
Tout bouge autour de moi de Dany Laferrière au crible de l'éthique décoloniale de Fritta Caro
}

\author{
Alexandre Beaudoin Duquette \\ Université du Québec à Montréal
}

\section{Introduction}

Dans Tout ce qu'on ne te dira pas, Mongo, Dany Laferrière écrit que « le racisme est un contact de deux pôles opposés sans que la lumière ne jaillisse » (227). Si la lumière se veut une métaphore de la connaissance, le racisme selon Laferrière correspondrait à une dialectique stérile : il y a contradiction sans pour autant qu'il émerge de celle-ci un savoir quelconque. Parmi les éléments qui font obstacle à l'émergence de cette connaissance, on trouve les stéréotypes. Ceux-ci stérilisent cette rencontre entre deux pôles opposés. Bref, ils constituent un obstacle à l'apprentissage et à la construction de savoir.

Les stéréotypes se constituent comme une représentation fictive et simplifiée du monde destinée à s'incruster de façon massive dans l'inconscient des individus afin de se substituer à ses représentations collectives. ${ }^{1}$ Ils impliquent également l'utilisation de la censure, entendue par Walter Lippmann, celui qui a donné au mot "stéréotype » le sens qu'on lui connaît aujourd'hui, comme l'action de séparer les individus des faits afin de contrôler l'interprétation qu'ils en font. Plus exactement, Lippmann soutient que la censure correspond à l'établissement d'une « barrière entre le public et l'événement» (chap. II.3). ${ }^{2}$ Les stéréotypes sont donc le fruit de la propagande entendue dans un sens large comme un « effort cohérent et de longue haleine pour susciter et infléchir des événements dans l'objectif d'influencer les rapports du grand public avec une entreprise, une idée ou un groupe » (Bernays, Propaganda 15).

Le racisme fait avorter une possibilité de connaissance. Il implique donc la méconnaissance de l'autre, l'ignorance par rapport à l'autre, un refus de

\footnotetext{
${ }^{1}$ Je renvoie ici à la conception durkheimienne des représentations collectives, qui les entend comme étant «le produit d'une immense coopération qui s'étend non seulement dans l'espace, mais dans le temps » (Durkheim 26). En ce sens, les représentations collectives sont le fruit d'un processus par lequel «une multitude d'esprits divers ont associé, mêlé, combiné leurs idées et leurs sentiments » et que «de longues séries de générations y ont accumulé leur expérience et leur savoir» (idem). Il s'agit donc d'« une intellectualité très particulière, infiniment plus riche et plus complexe que celle de l'individu » (idem). C'est pour cela qu'il faut le distinguer du stéréotype qui se propage de façon massive, ce qui implique une conception du public comme une agglomération d'individus.

2 Traduction personnelle de l'anglais.
} 
connaitre et de reconnaitre l'autre comme soi-même. La subordination de celuici se trouve justifiée de façon fictive par une vision du monde stéréotypée qui nous amène à simplifier son identité, à le caricaturer, à le prendre en pitié ou à le percevoir comme une menace. En ce sens, on délégitime son existence et on cherche à proscrire son action sur le monde. C'est dire que l'on nie son agentivité, le fait qu'il est un sujet et un acteur social.

En le subordonnant, on le déshumanise par le biais d'une méthode darwinienne simpliste. Le mot "race» renvoie ainsi au champ lexical de la zoologie. Le racisme, comme le définit Colette Guillaumin, consiste donc en « la croyance en l'hétérogénéité absolue de l'autre », c'est-à-dire, en la " différence de l'autre en tant que groupe tout en niant la surgence de l'individu au sein du groupe » (79). En d'autres mots, c'est se croire plus humain que l'autre et attribuer cette carence d'humanité à tout le groupe auquel on lui prête une appartenance. Celui-ci devient même la cause de cette prétendue carence d'humanité.

Le sociologue péruvien Aníbal Quijano affirme que «l'idée de race est, sans aucun doute l'instrument de domination sociale le plus efficace inventé ces 500 dernières années » et que celle-ci est le «produit du tout début de la formation de l'Amérique et du capitalisme ». C'est sur cette notion que se fonderait «l'eurocentrage du pouvoir mondial capitaliste et la distribution mondiale du travail et des échanges qui en découle » (111).

Par conséquent, le racisme est un problème dont la source se situe bien audelà de la conjoncture : il est apparu comme un instrument de domination qui a permis l'installation du système économique global en place. Il continue à biaiser la perception que nous avons du monde. La simple absence de doute quant à l'idée que les inégalités sociales sont le fruit d'une méritocratie est le résultat de la construction du métarécit de la modernité dont le but était de justifier l'instauration un système-monde capitaliste par des processus de colonisation (Lander). Ainsi, le «rayonnement» de la civilisation occidentale est attribué à ses «lumières", alors que les atrocités commises par le biais de son expansion coloniale sont perçues comme de simples accidents de parcours ou même le prix à payer pour que les peuples qui passent sous son rouleau compresseur jouissent du « développement» (Escobar).

L'infériorisation de l'autre ou l'idée que celui-ci est absolument différent de soi, qu'il correspond à une autre « race » - alors que, comme l'affirme Quijano, « il s'agit d'une construction idéologique nue, qui n'a, littéralement, rien à voir avec la structure biologique du genre humain et tout à voir, en revanche, avec l'histoire des rapports de pouvoir dans le capitalisme mondial, 
colonial/moderne et eurocentré » (113) -, implique une représentation fictive et simplifiée destinée à être incrustée, figée, de façon massive dans l'inconscient des individus, le stéréotype (Lippmann). En s'assemblant, les stéréotypes se constituent comme :

[...] une image ordonnée et plus ou moins cohérente du monde à laquelle nos habitudes, nos goûts, nos capacités, notre confort et nos espoirs se sont ajustés. Ils ne constituent peut-être pas une image complète du monde, mais ils correspondent à une image d'un monde possible auquel nous nous sommes adaptés. Dans ce monde, les gens et les choses occupent une place bien connue et la plupart de leurs actions correspondent à nos attentes. En ce lieu, nous nous sentons chez nous. Nous y avons notre place. Nous en sommes membres de plein droit. Nous savons comment il fonctionne. Nous y retrouvons également le charme de ce qui nous est familier, normal et fiable ; ses sillons et ses formes se situent là où nous sommes habitués à les trouver. Et bien que nous ayons renoncé à de nombreuses tentations avant de nous blottir dans ce moule, une fois que nous nous y sommes fermement installés, nous nous y sentons aussi confortables que dans une vieille paire de chaussures (chap. VII.1).

Le racisme est donc un comportement symptomatique d'une vision stéréotypée du monde. En ce sens, il est le fruit de ce que Walter Lippmann dénomme « censure », «le facteur clé qui limite l'accès [des hommes] aux faits" (chap. I.6). Il s'abreuve de cette image qui sépare l'individu du monde dans lequel il agit :

[...] nous devons remarquer un facteur en particulier. Il s'agit de l'insertion d'un pseudoenvironnement entre l'homme et son environnement. Le comportement de celui-ci est une réponse à ce pseudo-environnement. Cependant, précisément parce que c'est ${ }^{3}$ un comportement, les conséquences, si elles sont des actions, n’opèrent pas dans le pseudoenvironnement où le comportement est stimulé, mais dans l'environnement réel, là où les actions adviennent (chap. I.3)

Les stéréotypes sont donc des fabrications qui se substituent aux représentations collectives, et qui rendent les individus manipulables. L'idée est que, comme le soutient l'inventeur de la propagande Edward Bernays, si l'on contrôle l'image mentale du monde qu'ont les masses, on peut contrôler leurs attitudes : «le conseiller en relations publiques utilise parfois les stéréotypes courants, parfois il les combat et parfois il en crée de nouveaux » (Crystallizing Public Opinion, 162). ${ }^{4}$

La lutte contre les stéréotypes implique l'accès à des représentations du monde qui leur sont dissonantes. Dans le présent travail, j’explore certains éléments que nous offre la littérature de Dany Laferrière, qui peuvent contribuer à désarmer le stéréotype d'Haïti en tant que "pays maudit». Ainsi, non seulement on construit des outils pour désarmer une vision stéréotypée d'Haïti,

\footnotetext{
${ }^{3}$ Les cursives sont de l'auteur.

${ }^{4}$ Traduction personnelle de l'anglais.
} 
mais on crée également des possibilités d'apprentissage et de construction de savoir.

\section{Désarmer les stéréotypes, apprendre et connaître par la dissonance et l'art des diasporas}

Dans mes recherches précédentes, je démontrais que les œuvres et les témoignages des artistes latino-américains du Québec offraient des éléments pouvant contribuer à désarmer les stéréotypes de la propagande des institutions canadiennes liées à l'immigration. Pour ce faire, j'utilisais le concept de « dissonance.$^{5}$

Celui-ci renvoie à une notion inspirée d'un phénomène psychologique que Leon Festinger a baptisé « dissonance cognitive » au milieu du siècle dernier. Il s'agit d'un état psychologique qui survient lorsqu'un individu se trouve face à des éléments d'information incompatibles. Cette situation l'amènera à changer ses opinions ou son comportement afin de retrouver une vision du monde cohérente (93-102).

Lorsque nos stéréotypes sont mis en suspens par un état de dissonance cognitive, on se trouve face à la nécessité de se représenter le monde autrement. Ceci implique précisément une modification de notre opinion ou de notre comportement. Or, on suppose que l'apprentissage implique également « une modification du comportement résultant de l'expérience préalable » (Ardila 4). ${ }^{6}$ C'est donc dire que cette démarche mène à une possibilité d'apprentissage ou " pédagogique ». En travaillant sur les œuvres et les témoignages d'artistes latinoaméricains établis au Québec, j'ai remarqué que l'on y trouvait souvent des méthodologies et des concepts que l'on pourrait certainement utiliser dans les sciences sociales. Ceux-ci se constituent donc comme des possibilités de construction de savoir ou «épistémologiques ».

Lors des recherches qui m'avaient mené à la rédaction de ma thèse doctorale, une entrevue avec l'artiste visuelle Helena Martín Franco m'avait particulièrement inspiré. Elle affirmait que :

[...] l'instabilité que vit l'immigrant lorsqu'il arrive fait en sorte qu'il n'a rien à perdre, ce qui lui permet de trouver de nouveaux chemins parce qu'il doit chercher d'autres façons de vivre. Par conséquent, il doit se réinventer; il doit s'exposer; il doit prendre des risques et c'est un peu ce que l'artiste vit dans son atelier ou avec sa création. Une fois qu'il trouve des solutions, la création finit par se répéter, ce pour quoi l'artiste doit passer par

\footnotetext{
5 J'aborde ce problème dans plusieurs travaux. Les informations de quelques-uns d'entre eux sont disponibles dans la bibliographie du présent texte.

${ }^{6}$ Traduction personnelle de l'espagnol.
} 
des phases d'instabilité dans lesquelles il perd tout pour, à partir de cela, créer à nouveau (Propaganda migratoria canadiense 220). ${ }^{7}$

Le processus qu'elle décrit correspond précisément à ce que j'envisage comme la dissonance cognitive en tant que possibilité d'apprentissage et de construction de savoir. Cette situation correspond au moment où nous éprouvons le besoin de redonner de la cohérence à notre vision du monde lorsque nous nous trouvons face à des éléments d'information qui sont incompatibles avec celle-ci (Festinger 95). Alors, on se rend compte qu'il existe une incohérence entre ce que Walter Lippmann dénomme notre pseudoenvironnement et l'environnement réel. Nos stéréotypes se retrouvent donc en suspens et on a l'impression de tout perdre :

Les systèmes de stéréotypes peuvent être le cœur de notre tradition personnelle, les remparts de notre position dans la société. [...]

Il n'est donc pas surprenant que toute perturbation des stéréotypes nous apparaisse comme une attaque aux fondations de l'univers. De telles perturbations constituent une attaque aux fondations de notre univers et, en présence de grands enjeux, nous n'admettons pas facilement qu'il existe une distinction entre notre univers et l'univers. [...] Ils sont la garantie du respect de soi; ils sont la projection sur le monde du sens que nous attribuons à nos valeurs, à notre position et à nos droits. Les stéréotypes sont donc fortement imprégnés des sentiments qui leur sont attachés. Ils sont la forteresse de notre tradition et, derrière leurs remparts, nous continuons de nous sentir en sécurité dans la position que nous occupons (Lippmann chap. VII.1)

Assumer la dissonance cognitive constitue donc une prise de risque : cela implique une reconfiguration de notre identité, de notre place dans le monde. Cependant, il faut tenir compte de l'aspect solide, figé et fixe du stéréotype. N'oublions pas que ce qui caractérise le stéréotype, comme l'indique son suffixe qui provient du grec «stereos », c'est avant tout sa solidité, sa propension à se fixer et à figer notre vision du monde (Ewen et Ewen 4).

Désarmer les stéréotypes par la dissonance est donc une opération qui exige du tact, puisque, autrement, on se retrouve à pilonner les fondations de l'univers de l'apprenant; à attaquer une garantie de son amour propre. On le jette donc littéralement dans le vide : «Le tact permet de rester à distance. Il évite ce qui est choquant, la proximité trop grande par rapport à la personne et les blessures que l'on infligerait à son intimité » (Gadamer 30).

En ce sens, l'art apparaît comme une source de possibilités épistémologiques et pédagogiques intéressantes parce qu'il permet de conserver

\footnotetext{
7 Traduction personnelle de l'espagnol.
} 
cette distance par le biais d'une approche indirecte à la dissonance cognitive. Il est donc possible qu'il se constitue comme un objet susceptible de provoquer l'étonnement de celui qui le manipule et l'amener à l'interroger, à spéculer, à entreprendre un dialogue avec celui-ci afin de comprendre le monde autrement. L'art peut ainsi se transformer en une médiation, en un lien entre le sujet et le monde extérieur, en «frontière dissonante» plutôt qu'en «frontière stéréotypée ». ${ }^{8}$

À Montréal, le collectif L'Araignée, un regroupement d'artistes visuelles féministes qui réalisent des interventions urbaines et performatives dans les espaces publics, incorpore la dissonance cognitive à son approche. On peut citer en exemple Kamissa Ma Koïta ainsi qu'Helena Martín Franco.9 Lors d'une intervention intitulée $A l t e r o(s)$ philie, cette dernière a distribué un tutoriel qui offre des pistes pour développer une méthodologie d'apprentissage et de construction de savoir qui tiendrait compte de la dissonance cognitive. Notons que certaines étapes de la démarche qu'elle propose peuvent se constituer comme des éléments éthiques d'une méthode scientifique pour mener à bien un travail de terrain en sciences sociales. Car c'est de « vivre l'altérité » dont il s'agit (Martín Franco); une aspiration fondamentale de l'anthropologie.

Par le biais de la métaphore de l'haltérophile — qui, par le jeu des mots, des sens et des sons en vient à signifier «amour à l'autre » - son personnage de Fritta Caro, une immigrante devenue athlète de haut niveau afin de répondre aux attentes démesurées de sa société d'accueil, propose une démarche qui comprend, entre autres, les étapes suivantes :

- «La reconnaissance de se trouver sur des terres non cédées »

- «Se décentrer; se penser à la frontière, se penser soi-même une frontière »

- «Passer outre les stéréotypes »

- «Exercer l'écoute »

- «Prendre conscience de la dissonance cognitive »

8 Sur les concepts de «frontière dissonante » et de «frontière stéréotypée », voir Beaudoin Duquette, «Desarmar la frontera estereotipada desde el arte contextual de una colombiana en Quebec ».

9 Pour plus d'information sur le collectif l'Araignée, voir: http://daredare.org/fr/evenements/au-travers-de-incursions-gestes-et-postures (consulté le 18 août 2019). 
- «Éviter la condescendance »

- «Pratiquer la solidarité »

- «S'ouvrir à l'autre et par la suite s'ouvrir à l'autre en soi-même »

- $\quad$ Etre «attentifs aux discours d'inclusion qui semblent lutter contre les oppressions, mais qui en réalité les occultent et les facilitent : multiculturalisme, diversité, etc. »

- Démasquer « les rhétoriques qui cachent les logiques de subordination, d'oppression, de discrimination »

- Renoncer «à imposer votre version de l'autre sur l'autre »

- Soutenir « la visibilité d'autres mémoires qui ont été effacées par le récit unique de l'histoire officielle, point clé d'une bonne intégration »

- Raconter « amoureusement votre histoire et vos traditions »

- «Une sensibilisation aux traumas historiques hérités, des blessures coloniales, partagées ou pas, qui ne sont pas encore soignées »

- «Désobéir aux hiérarchies culturelles, raciales, de classe, de genre, d'origine, de croyances »

- Soutenir « la diffusion des pratiques de résistance à travers l'histoire face à la colonialité, l'eurocentrisme, le néolibéralisme, le capitalisme »

- «Entamer des rapports horizontaux d'échange et de respect réciproque »

- Donner « la voix et écoutez» $(i d e m)$.

Mon intention n'est guère de suivre pas à pas les étapes de la méthode proposée par Helena Martín Franco par le biais de son personnage de Fritta Caro. Celles-ci se trouvent plutôt en filigrane dans le contrepoint que j'effectue entre Tout bouge autour de moi et certains exemples de discours qui projettent sur Haïti le stéréotype de « pays maudit». Je fais donc référence au tutoriel d'Altero(s) philie de façon aléatoire, au fur et à mesure que celui-ci s'avère pertinent. J'espère ainsi explorer les possibilités épistémologiques de l'œuvre d'une artiste 
colombienne établie au Québec pour développer une analyse comparée entre deux discours dissonants. Dans le présent texte, j'explorerai particulièrement de quelles façons l'essai Tout Bonge autour de moi de Dany Laferrière peut nous amener à désarmer le stéréotype d'Haïti comme "pays maudit ». Je tente donc une expérience, c'est-à-dire que le présent texte constitue un essai : il s'agit de lire la relation dissonante entre le stéréotype du " pays maudit» et l'essai Tout bonge autour de moi au crible de l'éthique méthodologique de Fritta Caro afin d'explorer des possibilités d'apprentissage et de construction de savoir de l'art latino-américain et caribéen du Québec.

\section{La cascade de malheurs}

Dans Tout bouge autour de moi, Dany Laferrière raconte qu'une question qui lui a été posée par "une journaliste de la télé canadienne » alors qu'il se trouvait à Port-au-Prince dans les jours qui ont suivi le séisme de 2010. Cette question lui aurait fait voir "poindre un nouveau label qui s'apprête à nous enterrer complètement : Haïti est un pays maudit » (54) :

Je sais que certains l'emploient de bonne foi, ne trouvant d'autres termes pour qualifier cette cascade de malheurs. Ce n'est pas le bon mot, surtout quand on peut constater l'énergie et la dignité que ce peuple vient de déployer face à l'une des plus difficiles épreuves de notre temps. Mais chaque jour qui passe rend la tâche plus rude. Il suffit qu'une personne lance le mot «malédiction» sur les ondes pour qu'il se métastase comme un cancer. Avant qu'on se mette à parler de vaudou, de sauvagerie, de cannibalisme, de peuple de buveurs de sang, je me sens encore assez d'énergie pour contrer ça (55).

La remarque de Laferrière est fondée : en plus de l'anecdote racontée par l'auteur dans Tout bonge autour de moi, il n'est pas ardu de trouver des exemples d'utilisation de cette étiquette pour parler de la situation d'Haïti. Par exemple, sur le site de France 24, une chaîne de nouvelle en continu, on peut trouver un article datant des jours qui ont suivi le séisme de 2010 qui s’intitule « Haït, pays maudit » (France 24). On y justifie l'emploi de ce qualificatif précisément en énumérant des événements qui constitueraient une "cascade de malheur»: «Instabilité politique, misère, révoltes sociales, succession de cyclones... Le pays n'en est pas à sa première épreuve » (idem).

Quelques années plus tard, à la suite de l'ouragan Matthew, c'était le Journal de Montréal qui en rajoutait en publiant sur son site internet «une plongée interactive en cinq actes, comme si vous y étiez » (Meunier) dont le titre était, en créole pour en souligner encore mieux l'évidente et originelle validité proclamée, «Peyi madichon (HAÏTI PAYS MAUDIT) ${ }^{10}$ » (Daoust). Encore une fois, on justifie l'emploi de ce terme en renvoyant à une « cascade de malheur » : «Les

${ }^{10}$ En majuscule dans le texte original. 
insulaires du petit pays — surnommé la Perle des Antilles — sont pourtant constamment frappés par des catastrophes naturelles et problèmes politiques, autant de malédictions qui empêchent le pays de se relever» (Daoust). L'utilisation de la langue créole dans le titre du reportage « immersif » constitue également une justification de l'utilisation du label de « pays maudit » :

Cette succession de malheurs a d'ailleurs fait en sorte que le titre de notre reportage multimédia s'est un peu imposé de lui-même : « Ayiti peyi madichon », en créole, qui se traduit par « Haïti pays maudit ».

Un titre qui témoigne aussi humblement de notre volonté de laisser la parole au peuple haïtien (Daoust).

Ce à quoi le texte de Laferrière semble répondre : «Faut être vraiment désespéré pour accepter le mépris de l'autre sur soi ». Même si, affirme l'auteur, «certains l'emploient de bonne foi » (54-55).

Sans nécessairement remettre en question la «bonne foi » de l'équipe du Journal de Montréal, on peut à tout le moins remarquer sa maladresse. Car autant dans le reportage que dans le texte faisant la promotion de celui-ci, on parle d'un séjour " au pays de Laferrière » (Daoust et Meunier). On prétend donc « exercer l'écoute » et on suppose également une certaine " pratique de solidarité » (Martín Franco) par le choix d'un titre «qui témoigne humblement de notre volonté de laisser la parole au peuple haïtien » (Daoust). Cependant, on semble ignorer le combat contre le label de " pays maudit » que mène l'écrivain auquel on associe Haïti dans l'article.

Laferrière voit donc son œuvre désublimée par le stéréotype du «pays maudit». En effet, il semble que le mot «malédiction » "se métastase comme un cancer» menaçant ainsi «d'enterrer » son œuvre. Mais le fragment de Tout bonge autour de moi nous permet de diagnostiquer la "métastase». Il faut maintenant la combattre, "passer outre le stéréotype » de pays maudit et « prendre conscience de la dissonance cognitive » (Martín Franco.).

\section{Démasquer le « bon samaritain »}

Dans Tout ce qu'on ne te dira pas, Mongo, après avoir décrit le racisme comme une « rencontre entre deux pôles opposés sans que la lumière jaillisse » (227), Laferrière propose l'exercice suivant : "Il se peut que vous soyez témoin d'un acte public de racisme. Ne soyez pas le premier à le dénoncer. Laissez la possibilité à un natif de le faire. Le racisme, c'est l'affaire de tout le monde » (226). Propager le stéréotype d'Haïti comme étant un «pays maudit » implique l'action de reproduire, de nourrir et de renforcer une vision réductrice et erronée de l'endroit et de ceux qui le peuplent ainsi qu'une fiction construite par 
l'Occident afin de justifier sa domination coloniale comme étant naturelle. Il semble donc logique de suivre la stratégie de Laferrière qui propose que «ce terme ne [puisse] être combattu que là où il a germé : dans l'opinion occidentale » (Tout bouge autour de moi 55).

Dans le sous-titre qui accompagne l'article faisant la promotion du reportage immersif sur Haïti à la suite du passage de l'ouragan Matthew, on rapporte « le cri du cœur [d'une] sinistrée de Kafou Diri »: "Mes amis aidez-nous! On a besoin d'un endroit pour se loger! Vous qui êtes de bons samaritains, venez nous aider! » (Meunier)

Ces paroles décontextualisées semblent interpeller le lecteur plus que le journaliste et, bien qu'elles puissent avoir pour intention de le sensibiliser à la détresse provoquée par le passage de Matthew, elle renforce également le stéréotype de l'Occident jouant le rôle du «bon samaritain ». Sans nécessairement exercer du mépris, le document affiche néanmoins une sorte de «supériorité bienveillante» (Robert 323) qui laisse un parfum de condescendance, ce que Fritta Caro recommande précisément d'éviter dans sa pratique de la solidarité.

De plus, comme l'affirme Teun A. van Dijk, «les citations constituent une stratégie puissante permettant au journaliste d'éviter des contraintes liées à l'impersonnel, aux opinions, aux points de vue et aux formalités » (76). Dans le cas présent, en plaçant cet appel à l'aide fait à l'équipe du Journal de Montréal par des sinistrés comme sous-titre d'un article faisant la promotion d'un reportage, on lui donne de nouveaux sens. D’un côté, on souligne «notre impuissance » qui nous est « renvoyée en plein visage » (Meunier). Le «bon samaritain » peut donc se déresponsabiliser. Le mieux qu'il puisse faire, c'est de sentir de la compassion.

Mais il y a également la construction du stéréotype d'Haïti comme " pays maudit» qui se perpétue et qui est nourri par celui du «bon samaritain» occidental. Ainsi, l'Occident se déculpabilise par sa bienveillance soudainement reconnue par des paroles rapportées : « on voudrait bien, mais que pouvonsnous faire? »

La réponse ne saurait tarder. Quelques lignes plus loin, on peut lire : « $\mathrm{A}$ travers une série de vidéos immersives, de plans aériens impressionnants, de photos spectaculaires et de rencontres inspirantes, découvrez la Perle des Antilles 
comme vous ne l'avez encore jamais vue » (Meunier. $)^{11}$. Et pourtant, celle-ci est dépeinte une fois de plus comme « un pays maudit».

La lecture de Tout bonge autour de moi peut nous amener à questionner ce discours sur Haïti qui « semble lutter contre les oppressions, mais qui en réalité les occultent et les facilitent» et qui «cache les logiques de subordination» (Martín Franco). Si l'on « exerce l'écoute » de la voix narrative de Laferrière, on notera que l'auteur pourrait nous inviter à nous méfier. Car...

Ce sont des images si puissantes qu'elles cachent le reste. Comment font-ils ce choix? Ces images sont-elles naturellement accrocheuses ou est-ce la répétition qui nous les rend familières? Je sens qu'on est en train de nous confectionner une mémoire. Ce sont souvent les dernières qu'on voit avant de s'endormir. Ce choix d'images s'est-il fait par hasard ou ces réalisateurs savent-ils d'expérience ce qui touchera le public (Tout bouge autour de moi 61). ${ }^{12}$

Cette réflexion rappelle certaines caractéristiques du processus de construction du stéréotype : l'image, la répétition, la confection d'une mémoire, l'inconscient (qui fait surface par le biais du rêve lorsqu'on est endormi) et le public. Tous ces éléments sont traités dans Public Opinion de Walter Lippmann, l'œuvre qui donna naissance à ce concept.

Ici, l'écrivain ne se contente pas d'évoquer; il nous offre des outils d'analyse critique du discours des médias de masse. Le reportage immersif du Journal de Montréal, d'abord avec son titre, «Haiti, pays maudit », ensuite avec la répétition d'images de dévastation, peut consolider le stéréotype dans notre inconscient.

Les photos et les vidéos qui accompagnent le reportage n'offrent pratiquement aucun autre paysage que celui de la dévastation. Et les mots " chaos », « angoisse », " vision apocalyptique » provoquent l'impression que ce que Lippmann dénomme le «monde extérieur» est une « confusion rayonnante et bourdonnante », ce pourquoi nous préférerions peut-être nous en tenir à «ce que notre culture a déjà défini à notre place » (Lippmann chap. VI.1).

Nous nous trouvons en présence de ce qu'Herbert Marcuse qualifie de « formules hypnotiques qui, en se répétant sans cesse, fixent la signification dans l'esprit du destinataires » (94). ${ }^{13}$ Ainsi, la répétition de l'association entre « pays maudit » et « Haïti » « des images qui collent à l'esprit et au produit » est établie et celle-ci « s'impose avec une écrasante concrétude » (idem). C'est peut-être pour cela que Dany Laferrière craint que cette étiquette «nous enterre complètement» (54).

\footnotetext{
${ }^{11}$ C'est moi qui souligne le verbe à l'impératif.

${ }^{12}$ C'est moi qui souligne.

13 Traduction personnelle de l'anglais.
} 
Ainsi, l'image du « pays maudit» se cristallise et il est de plus en plus difficile pour l'entité qui la subit de s'en défaire. Comme le soutient Helena Martín Franco dans son tutoriel, on «impose notre version de l'autre sur l'autre ». L'Occident, pour sa part, hérite du beau rôle, celui de « bon samaritain » et, bien qu'elle renvoie à son public l'image de sa propre impuissance, sa domination symbolique sur l'Autre, sa colonialité sur Haïti, se réaffirment. Le stéréotype de « pays maudit » renvoie donc à une attitude inévitablement condescendante de la part du «bon samaritain » :

Celui qui vous aide s'accorde parfois le pouvoir de vous juger. C'est la moindre des choses que de l'écouter. L'argument est simple : votre savoir-faire a échoué. Et ne vous avisez pas de protester, car c'est vrai. C'est lui qui aide et non le contraire. Et il vous met sa culture sous le nez. Tout cela dit sur un petit ton de fausse humilité, ce qui est la pire des vanités. Et l'orgueil de croire que l'autre n'a pas compris la situation. Et qu'il a avalé votre jeu. On devrait donner un petit cours de culture populaire à ceux qui viennent aider... (Tout bonge autour de moi 119-120)

En lui mettant «sa culture sous le nez», il impose également sa propre version de l'histoire et, ce faisant, il efface celle de l'autre (54). Laferrière synthétise ce processus de la façon suivante :

Pendant longtemps, Haïti a été vu comme la première république noire indépendante du monde, et la deuxième en Amérique après les États-Unis. Cette indépendance ne nous [a] pas été accordée entre deux martinis, des sourires hypocrites et des discours pompeux sur une pelouse couverte de confettis; elle a été conquise de haute lutte à la plus grande armée européenne, celle de Napoléon Bonaparte. Mon enfance fut bercée par des histoires d'esclaves qui n'avaient pour toute arme que leur désir de liberté et une bravoure insensée. Ma grand-mère me racontait, les soirs d'été, les exploits de nos héros qui devaient tout prendre à l'ennemi : les armes comme les techniques de combat. Même la langue française fut « un butin de guerre ». Et brusquement, vers la fin des années 1980, on a commencé à parler d'Haïti uniquement en termes de pauvreté et de corruption (54).

En effaçant l'histoire de la «première république noire indépendante du monde » ou en la résumant à une cascade de malheur, on dérobe au peuple haïtien son agentivité. Ainsi, on arrive à voir l'amnésie comme une solution. À ce sujet, Laferrière attire l'attention sur l'idée de certains de concevoir 2010 comme «l'année zéro » :

J'ouvre la télé, tôt ce matin, pour tomber sur cet analyste politique qui croit qu'Haïti pourrait repartir d'un bon pied s'il consent à oublier tout ce qui a précédé le séisme. On évoque un moment la situation d'avant qui n'était pas bien reluisante. La scène est assez choquante en elle-même puisque l'analyste et le journaliste qui l'interviewe sont confortablement assis tandis que derrière (plein écran) on voit défiler les images de désolation. Il suffit de regarder ces scènes d'horreur (des bouches hurlant sans qu'on entende un son) pour acquiescer à tout ce qui se dit. Cette technique d'intimidation est si généralisée qu'on n'y voit rien d'anormal. En fait, on nous présente un problème tout en nous empêchant de réfléchir. La réponse est derrière la question. Pour ramasser tout cela en un seul vocable supposément riche d'espoir, l'expert interviewé avance : « année zéro ». Zorro est arrivé. C'est la première fois que j'entends évoquer, à l'encontre d'Haïti, 
ce concept d'année zéro. Je n'arrive pas à avaler cette idée, malgré ces images insupportables qui me déchirent la rétine (58-59).

Lorsque l'on parcourt l'internet, on s'étonne de la quantité d'entrées qui font mention de 2010 comme «l'année zéro » pour Haïti. Il semble que la formule s’intègre de façon passablement logique au stéréotype du « pays maudit » : pour mettre fin à la malédiction, il suffirait de faire table rase. On se retrouve donc en présence de deux sophismes qui, en se complétant, donnent l'impression de constituer à la fois le diagnostic d'une réalité intolérable et son chemin vers l'utopie. ${ }^{14}$ La simplification de la réalité haïtienne est donc ici prodigieuse. Elle traverse le temps. Elle se constitue comme un univers parallèle autonome doté de sa propre histoire et de son propre devenir : la « cascade de malheur », puis, «l'année zéro » qui permet d'espérer des jours meilleurs en effaçant le passé marqué par la « malédiction ». Laferrière argumente cependant qu'Haïti n'est pas un pays frappé par un «malheur auquel on semble voué par la destinée, par le sort » (Robert 1031) :

Mon seul argument: Qu'a fait de mal ce pays pour mériter d'être maudit? Je connais un pays qui a provoqué deux guerres mondiales en un siècle et proposé une solution finale et on ne dit pas qu'il est maudit. Je connais un pays insensible à la détresse humaine, qui n'arrête pas d'affamer la planète depuis ses puissants centres financiers et on ne le dit pas maudit. Au contraire, il se présente comme un peuple béni des dieux, plutôt de Dieu. Alors pourquoi Haiti serait-il maudit? (Tout bonge autour de moi 55)

La réflexion de Laferrière nous amène vers des épistémologies du Sud. Elle n'est d'ailleurs pas sans rappeler les questions posées par Eduardo Galeano au sujet de l'Amérique latine :

L'Amérique latine est-elle une région du monde condamnée à l'humiliation et à la pauvreté? Condamnée par qui? La faute de Dieu, la faute de la nature? Le climat suffocant, les races inférieures? Le malheur ne serait-il pas un produit de l'histoire faite par les êtres humains et qui peut, par conséquent, être défait par ceux-ci? (340)

Ainsi, l'essai de Laferrière laisse entrevoir ce qu'Helena Martín Franco décrit par le biais de Fritta Caro comme « des pratiques de résistance à travers l'histoire face à la colonialité [et] l'eurocentrisme ». Une histoire de résistance et de colonialité que le stéréotype du «pays maudit » contribue à oblitérer. Laferrière rappelle qu'il est impossible d'effacer l'histoire, car elle est inscrite dans les représentations collectives de la population :

Le séisme n'a pas détruit Port-au-Prince, car on ne pourra construire une nouvelle ville sans penser à l'ancienne. Le paysage humain compte. Et sa mémoire fera le lien entre l'ancien et le nouveau. On ne recommence rien. C'est impossible d'ailleurs. On continue. Il y a des choses qu'on ne pourra jamais éliminer d'un parcours : la sueur humaine. Que fait-on de ces deux siècles, et de tout ce qu'ils contiennent, qui ont précédé l'année zéro?

\footnotetext{
${ }^{14}$ Ce résumé de la structure du genre littéraire de l'utopie est inspiré de la description qu'en fait Horacio Cerutti Guldberg (50-51).
} 
Les jette-t-on à la poubelle? Une culture qui ne tient compte que des vivants est en danger de mort. (Tout bouge autour de moi 59).

Le passé existe. Il nous habite. La mémoire façonne les représentations collectives. C'est pour cela que c'est cette mémoire qui est en jeu : «Je sens qu'on est en train de nous confectionner une mémoire ", dit Laferrière (61). C'est précisément par ce processus que les stéréotypes travaillent à désarmer les représentations collectives, c'est-à-dire :

[...] le produit d'une immense coopération qui s'étend non seulement dans l'espace, mais dans le temps; pour les faire, une multitude d'esprits divers ont associé, mêlé, combiné leurs idées et leurs sentiments; de longues séries de générations y ont accumulé leur expérience et leur savoir. Une intellectualité très particulière, infiniment plus riche et plus complexe que celle de l'individu, y est donc concentrée (Durkheim 26)

Si l'on accepte la version occidentale de l'histoire haïtienne diffusée à une échelle massive, qui se résume à une " cascade de malheur » ou à une «malédiction », on nie nos représentations collectives. En acceptant « la version de l'autre sur soi » (Martín Franco), on intègre la malédiction que l'autre projette sur nous; on « accepte le mépris de l'autre sur soi » (54). Car l'autre a passé notre histoire sous silence :

Au moment de l'Indépendance, le monde occidental s'est détourné de cette nouvelle république qui a dû savourer seule son triomphe. Tel était le destin de ce peuple qui venait de sortir du long tunnel noir et gluant de l'esclavage. L'Occident a toujours refusé de reconnaitre cette arrivée au monde. L’Europe comme l'Amérique lui ont tourné le $\operatorname{dos}(64-65)$.

C’est ici que Dany Laferrière nous invite à aller au-delà de sa propre écriture.

\section{Voir Haïti autrement}

Ce qu'Edgardo Lander et al. ont dénommé «la colonialité du savoir» contribue à l'eurocentrage de la connaissance, reléguant ainsi les épistémologies provenant d'autres lieux d'énonciation que ceux situés en Occident au rang de cosmovisions périphériques. L'œuvre de Laferrière peut constituer une porte d'entrée pour un large public à une épistémologie qui lui serait peut-être méconnue.

En ce sens et comme je l'ai mentionné plus haut, ses œuvres peuvent soutenir "la diffusion des pratiques de résistances à travers l'histoire face à la colonialité [et] l'eurocentrisme» (Martín Franco). Dans Tout bouge autour de moi, une multitude de figures importantes apparaissent au fil des récits, ce qui pourrait inspirer la construction d'un corpus d'auteurs, d'intellectuels et d'artistes liés à Haïti. Ainsi, l'écriture de Laferrière nous offre des armes pour contrer l'imposition du «label de pays maudit», mais également contre la colonialité et 
l'eurocentrage du savoir en attirant l'attention sur la contribution haïtienne à la culture universelle.

Ainsi, Port-au-Prince accueille un important salon du livre et Haïti inspire André Malraux, Paul Morand et Graham Green. On rencontre également l'écrivain et peintre Frankétienne, l'écrivain Rodney Saint-Éloi qui est également l'éditeur de Mémoire d'encrier ainsi que les intellectuels Georges et Mireille Anglade. On fait connaissance avec le locuteur de radio Compè Filo et le poète Christophe Charles. On nous mentionne plusieurs peintres comme Saint-Brice, Hector Hippolyte, Antonio Joseph, Cédor, Lazare, Philippe-Auguste, Wilson Bigault, Benoît Rigaud, Jasmin Joseph (95) ainsi que le poète Roussan Camille et l'anthropologue Jean Price-Mars...

Mais, si nous voulons revenir à cette mémoire haïtienne en dispute, Dany Laferrière nous offre une piste dans un chapitre qui s'intitule «La maison de Trouillot ». Il y raconte qu'alors qu'il se trouve en compagnie de l'écrivain Lyonel Trouillot, «le frère aîné des Trouillot fait son apparition dans un fauteuil ». Il s'agit de Michel-Rolph Trouillot. Laferrière nous le présente en nous expliquant qu'il est «l'auteur du premier livre d'histoire d'Haïti en créole, Ti dife boule sou istona Ayiti» et qu'il pose « un regard de marxiste » sur l'histoire haïtienne (38). Michel-Rolph Trouillot est également l'auteur de Silencing the Past: Power and the Production of History, un livre qui peut contribuer à notre compréhension de comment et pourquoi le stéréotype du " pays maudit » s'est érigé de façon aussi puissante.

Selon lui, « toute narration historique est un tissu de silences » et ces silences sont le résultat d'un " pouvoir inégal dans la production de sources, d'archives et de narrations » (27). ${ }^{15}$ Une façon éthique de raconter l'histoire consiste à la concevoir comme un " processus social ». Cela suppose l'étude d'une population donnée en envisageant ses membres en tant qu'agents, c'est-à-dire, des personnes qui occupent une position structurelle; en tant qu'«acteurs en constante relation avec un contexte » et en tant que sujets, « des voix conscientes de leur propre capacité d'énonciation » (aware of their vocality) (23).

Or, le récit de l'histoire haïtienne s'est construit sans tenir compte de ce principe : «En ce qui concerne l'historiographie haïtienne, tout comme dans le cas de la plupart des pays du tiers-monde, ces ententes préalables [sur lesquels reposent ces récits historiques] ont été profondément façonnées par les conventions et les procédés occidentaux ». Dans le cas de l'histoire haïtienne, cela serait principalement dû au fait que «l'écriture et la lecture de

\footnotetext{
15 Traduction personnelle de l'anglais.
} 
l'historiographie haïtienne impliquent la maîtrise et l'accès formel à une langue et une culture occidentales - principalement le français ", ce qui empêcha d'entrée de jeu la majorité des Haitiens de participer à sa production (55). Les conséquences de cette exclusion ne doivent guère être sous-estimées, surtout lorsqu'on tient compte du fait que la Révolution haïtienne a été menée par des esclaves.

Or, au moment où cet événement d'une importance fondamentale est survenu, les principaux acteurs de la Révolution haïtienne n'étaient perçus ni comme des agents, ni comme des acteurs, ni comme des sujets. Trouillot fait remarquer que cette conception a laissé des traces dans l'historiographie de la Révolution haïtienne :

[...] plusieurs historiens sont davantage disposés à accepter l'idée selon laquelle des esclaves ont pu être influencés par des blancs ou des mulâtres libres, même si l'on sait qu'ils n'avaient que des contacts limités avec ceux-ci, qu'ils ne le sont à accepter l'idée que des esclaves aient pu convaincre d'autres esclaves qu'ils ont le droit de se révolter (103).

En ce sens, la Révolution haïtienne était un événement «impensable» lorsqu'elle est survenue du point de vue de ceux qui détenaient « le pouvoir des archives » (archival power) (27) :

Lorsque la réalité ne s'accorde pas avec des croyances profondes, les êtres humains ont tendance à formuler des interprétations qui forcent l'entrée de cette réalité à l'intérieur du cadre de ces mêmes croyances. Ils conçoivent des formules afin de réprimer l'impensable et de le ramener à l'intérieur du domaine du discours accepté (72).

Autrement dit, la Révolution haïtienne a placé l'Occident en état de dissonance cognitive. Car « ce qui est survenu en Haïti contredit également la majeure partie de ce que l'Occident s'est racontée à la fois à soi-même et aux autres sur soi-même »(107). Comme l'affirme Leon Festinger, «[...] si une personne connait plusieurs choses qui ne sont guère psychologiquement cohérentes les unes avec les autres, elle essaiera de les rendre plus cohérentes de diverses manières » (94). ${ }^{16}$ Le réflexe de l'Occident face à cette situation qui mettait en échec la cohérence de son récit et de sa place dans le monde a été simplement de passer la Révolution haïtienne sous silence. On lui a «tourné le dos» comme le dit si bien Laferrière, «l'Occident a toujours refusé de reconnaitre cette arrivée au monde » (Tout bonge autour de moi 65).

Le stéréotype du " pays maudit » est le produit d'une histoire racontée sans tenir compte de ceux qui l'ont construite. Il est l'héritage du silence gardé par l'Occident sur la Révolution haïtienne parce que celle-ci contredisait les

\footnotetext{
16 Traduction personnelle de l'anglais.
} 
croyances racistes qui justifiaient le maintien de l'esclavage et de sa domination coloniale :

Alors que le pays avait été ostracisé durant la majeure partie du 19e siècle, sa situation s'est détériorée autant sur le plan économique que politique —en partie à cause de ce même ostracisme. Au fur et à mesure qu'Haïti déclinait, la réalité de la révolution semblait de plus en plus distante, une improbabilité qui s'était réalisée dans un passé gênant à laquelle personne n'avait d'explication rationnelle. La révolution qui était impensable est devenue un non-événement (Trouillot 98).

En expliquant la réalité haïtienne par une « cascade de malheurs » résultant d'une « malédiction », le stéréotype du « pays maudit» reproduit ce long silence de l'Occident à propos de la Révolution haïtienne. Et ce que l'Occident cherche à passer sous silence, c'est le rôle raciste et colonialiste qu'elle a joué dans l'histoire haïtienne : «Moins le colonialisme et le racisme paraissent importants dans l'histoire mondiale, plus la Révolution haïtienne semble également perdre de son importance» (98). L’idée de sort que renferme la notion de «malédiction » passe sous silence que les Haïtiens sont à la fois agents, acteurs et sujets de leur histoire et de leurs processus sociaux.

\section{Conclusion}

Le récit de Laferrière nous permet d'utiliser la littérature pour déjouer le pouvoir des archives en nous ouvrant la porte sur d'autres façons de raconter l'histoire haïtienne, que ce soit en nous faisant connaitre un important historien comme Michel-Rolph Trouillot ou simplement en rapportant les histoires que sa grand-mère racontait sur la Révolution haïtienne. Comme l'énonce Helena Martín Franco par son personnage de Fritta Caro dans le tutoriel Altero(s) philie, en plus de « raconter amoureusement son histoire et ses traditions », cela permet de semer des dissonances sur l'histoire officielle en nous fournissant des pistes pour « désobéir aux hiérarchies culturelles, raciales, de classe, de genre, d'origine, de croyance ».

En dénudant le pouvoir des archives, on accroît notre compréhension des racines colonialistes et racistes du stéréotype du pays maudit; on comprend ce qu'il passe sous silence : que les Haïtiens sont à la fois agents, acteurs et sujets de leur histoire. En ce sens, la littérature des Haïtiens en particulier et des LatinoAméricains en général qui sont établis au Québec constitue une possibilité d'« outrepasser les stéréotypes» hérités d'une vision du monde coloniale. La littérature nous permet d'entrer avec tact en dissonance cognitive, mais également de construire un nouveau savoir à partir de cet état en nous offrant d'autres possibilités de se représenter le monde. On passe ainsi du stéréotype, à la dissonance cognitive, à la fantaisie et finalement à la reconfiguration de notre comportement, de notre discours ou de notre perception. Cette méthodologie 
nous est inspirée d'une artiste colombienne établie au Québec, Helena Martín Franco dont le tutoriel constitue une expérience débouchant sur des possibilités épistémologiques qui méritent d'être explorées.

Les nombreux d'intellectuels et d'artistes qui défilent dans Tout bonge autour de moi nous placent devant une population qui contribue à la connaissance et à la culture universelle. La proposition d'Helena Martín Franco de « s'ouvrir à l'autre et par la suite s'ouvrir à l'autre en soi-même » nous amène à approfondir nos connaissances sur les figures mentionnées par Laferrière. En combinant l'écriture de Laferrière et la méthodologie proposée par Helena Martín Franco afin de "vivre l'altérité », on ouvre ainsi une voie pour contribuer au projet de Trouillot de « raconter l'histoire de l'Occident à nouveau d'une manière qui mette de l'avant la perspective du monde » (107). 


\section{Bibliographie}

Ardila, Rubén. Psicología del aprendizaje. Siglo XXI, 1979.

Beaudoin Duquette, Alexandre. Propaganda migratoria canadiense y arte latinoamericano en Montreal : un contrapunteo disonante. 2015. Universidad Nacional Autónoma de México, $\mathrm{PhD}$ dissertation.

Beaudoin Duquette, Alexandre. «Desarmar la frontera estereotipada desde el arte contextual de una colombiana en Quebec ». Alteridades, vol. 56, no. 28, 2018, pp. 35-46.

---. « Todo lo que no te diran, Mongo: Desarmando los Mythscapes Canadienses con la Literatura de Dany Laferriére ». De raíz diversa, vol. 5, no. 9, 2018, pp. 195226.

---. « Contrapunto y contradicción. Estereotipo y disonancia entre el rap latinoamericano en Montreal y la propaganda migratoria canadiense ». Algunas formas politicas y socioculturales de habitar espacios, edited by Lince Campillo et. al., Universidad Nacional Autónoma de México, 2018, pp. $107-$ 140.

---. «Multiculturalismo canadiense y arte colombiano en Montreal: desarmando estereotipos nacionales con Fritta Caro ». Revista Cultura y Representaciones Sociales, vol. 13, no. 26, 2019, p. 129-160.

Bernays, Edward. Crystallizing Public Opinion. Liveright Publishing corporation, 1961. ---. Propaganda: Comment manipuler l'opinion en démocratie. Lux, 2008.

Cerutti Guldberg, Horacio. «Lo útopico operante en la historia, como núcleo motriz de la praxis de la resistencia en Nuestra América ». Tópicos, edited by Martínez Contreras et. Al., Siglo XXI, 2007, pp. 49-55.

Daoust, Jean-Philippe. «AYITI Peyi madichon (HAITI PAYS MAUDIT)». Le Journal de Montréal, 2017. www1.journaldemontreal.com/2017/haiti/index.html. Accessed 18 Aug. 2019.

Durkheim, Émile. Questions préliminaires. Les formes élémentaires de la vie religieuse, vol. 1, Université du Québec à Chicoutimi, 2001.

Escobar, Arturo. Encountering Development: the Making and Unmaking of the Third World. Princeton University Press, 2012.

Ewen, Elizabeth and Stuart Ewen. Typecasting: On the arts \& sciences of human inequality. Seven Stories Press, 2006.

Festinger, Festinger. «Cognitive Dissonance ». Scientific American, vol. 207, no. 4, 1962, pp. 93-102.

Bertsch, Marina. « Haïti, pays maudit ». France 24, 13 Jan. 2010. www.france24.com/fr/20100113-ha-ti-pays-maudit. Accessed 18 Aug. 2019.

Gadamer, Hans-Georg. La philosophie herméneutique. Presses universitaires de France, 1996. 
Galeano, Eduardo. Las venas abiertas de América Latina. Siglo XXI, 2019.

Guillaumin Colette. L'idéologie raciste. Genèse et langage actuel. Institut d'études et de recherches interethniques et interculturelles, 1972, pp. 243-247.

Laferrière, Dany. Tout ce qu'on ne te dira pas, Mongoi. Mémoire d'encrier, 2015.

Lander, Edgardo (éditeur). La Colonialidad Del Saber: Eurocentrismo Y Ciencias Sociales : Perspectivas Latinoamericanas. Consejo Latinoamericano de Ciencias Sociales, 2000.

Lippmann, Walter. Public Opinion. Project Gutenberg, 2014, www.gutenberg.org/cache/epub/6456/pg6456-images.html. Accessed 18 Aug. 2019).

Meunier, Hugo. « Haïti comme si vous y étiez ». Le journal de Montréal, 11 Feb. 2017, www.journaldemontreal.com/2017/02/11/haiti-comme-si-vous-y-etiez. Accessed 18 Aug 2019.

Marcuse, Herbert. One-Dimensional Man. Routledge, 2007.

Martín Franco, Helena. Tutoriel Altéro(s)philie de Fritta Caro. Laboratoire L'araignée, 2018, laboratoire.laraignee.ca/tutoriel-alterosphilie-fritta-caro.pdf (consulté le 18 août 2019).

Quijano, Aníbal. " "Race” et colonialité du pouvoir ». Mouvements, vol. 51, no. 3, 2007, pp. 111-118.

Robert, Paul. Dictionnaire alphabétique \& analogique de la langue française. S.N.L., 1972.

Trouillot, Michel-Rolph. Silencing the Past: Power and the Production of History. Beacon Press, 2015.

Van Dijk, Teun A. News as discourse. Lawrence Erlbaum Associates, 1988. 